\title{
Predicting ESRD Risk via Supervised and Ensemble Machine Learning Technique
}

\author{
Nipa Sarkar ${ }^{1}$, Asha Rani Borah ${ }^{2}$ \\ Department of Computer Science ${ }^{1,2}$, New Horizon College of Engineering ${ }^{1,2}$ \\ Bangalore-560103, Karnataka, India \\ Email: nipas94@gmail.com ${ }^{1}$, asha.borah@gmail.com ${ }^{2}$
}

\begin{abstract}
Around 10\% population of the world gets affected by kidney disorders or chronic kidney disorders and almost millions of them die every year. If this trend continues then it is assessed that the total number of cases of kidney disorder will rise excessively since it is a global health crisis. By implementing Machine Learning techniques, it is possible to detect the risk of ESRD End-Stage Renal Disease) in the prior stage and proper medication can reduce the percentage of the people suffering from the renal disease risk. This paper introduces the ensemble machine learning algorithm to predict the ESRD risk and helps in fasten the decisionmaking process of the doctors. The main purpose of this paper is to observe the accuracy score of various supervised machine learning model and finally use the ensemble technique to get the highest accuracy for predicting the ESRD risk. The intention behind this paper is to build a a non-invasive machine learning model that can predict the ESRD risk by using ESRD dataset which is established by gathering data from various ESRD patients generated through IOT sensors.
\end{abstract}

Index Terms- ESSRD; IOT sensors; Supervised; Ensemble.

\section{INTRODUCTION}

End stage renal disease is nothing but the final stage $\left(5^{\text {th }}\right.$ stage) of the Chronic Kidney Disease. At this stage the patient's kidney function only $10 \%-15 \%$ of their normal capacity. When the functionality of the kidneys is this low, it cannot remove toxic waste or fluids effectively from the body. At this condition dialysis or kidney transplant is mandatory to make the person alive. But dialysis being complicated procedure to perform due to its complex nature of conduction to every patient. It is also a challenging process due to segregation of maintenance \& treatment structures.

Symptoms of ESRD are: 1) Nausea, Vomiting and Loss of appetite and Fatigue and weakness. 2) Sleep problems and Changes in the quantity of urine. 3) Decreased mental sharpness and Muscle twitches and cramps. 4) Swelling of feet and ankles, Persistent itching, Chest pain, if fluid builds up around the lining of the heart and Shortness of breath, if fluid builds up in the lungs, High blood pressure (hypertension) that's difficult to control.

In this paper we will be using machine learning algorithms to predict the early stage renal diseases. the ensemble classifier will help in predicting the disease early stage. This will help the patients to take corrective measures upfront and reduce the chances of kidney failures. The main purpose of the paper is to develop non-invasive approach that can predict the probability of occurring a complication for ESRD dialysis patient. Risk prediction accuracy is the frequency of predicting the right state of the patient. This paper also provides a detailed information on the literature survey, proposed features, system details and various machine learning algorithms which provide the accuracy score of predicting ESRD risk correctly.

\section{LITERATURE SURVEY}

Two main fields are taken into consideration for discussing the ESRD risks- (1) dialysis and (2) machine learning algorithms. An inclusive depiction of the existing technology is deliberated in the following subsections which are connected with this paper work.

\subsection{Dialysis Biomarkers}

Biomarkers are biological markers which is used to make suitable information available for analyzing several risks of a patient. The National Health Institutes defines biomarker as a distinguishing measurable indicator using which a particular biological processes, pathogenic processes, pharmacologic responses, severity or existence. The kidneys of human body preserve constituency and amount of liquids in the body via regulatory system and balances water level, electrolytes, acidity etc. and controls the elimination of contaminants and unsolidified.[4]

\subsection{Machine Learning Algorithms}

Machine learning is a domain that enables the system automatically to learn through experiences and improve performance by itself without being explicitly programmed by a developer. The main intention of machine learning is to allow the system/computers experience automatically without human involvement or support and perform actions consequently. Here, we will be discussing about supervised ML algorithms which can help to predict the early stage renal diseases. The ensemble techniques will help in predicting the risk of the disease in the early stage and help the doctor to improve quick decision making. This will help the patients in taking counteractive 
measures upfront and reduce the chances of kidney failures.

\section{IMPLIMENTATION STEPS}

The main objective is to:

1) Build a base line machine learning model that can predict the ESRD disease.

2) Validate model by using benchmarking models like Random Forest, Naïve Bayes, KNN to forecast the values.

3) Perform Grid search CV to identify the right set of parameters for the models.

4) Compare the accuracy of the models using $\mathrm{K}$ fold validation techniques.[1]

\section{SYSTEM DETAILS}

In the existing system, study is conducted on dialysis patient in ESRD. Patients are analysed in terms of biomarkers values. Based on datamining techniques, a modern data analytics environment is developed using python. The System design details describes about the models used for analysis, architecture required to build the models and the statistical concepts behind all the algorithms used.[1]

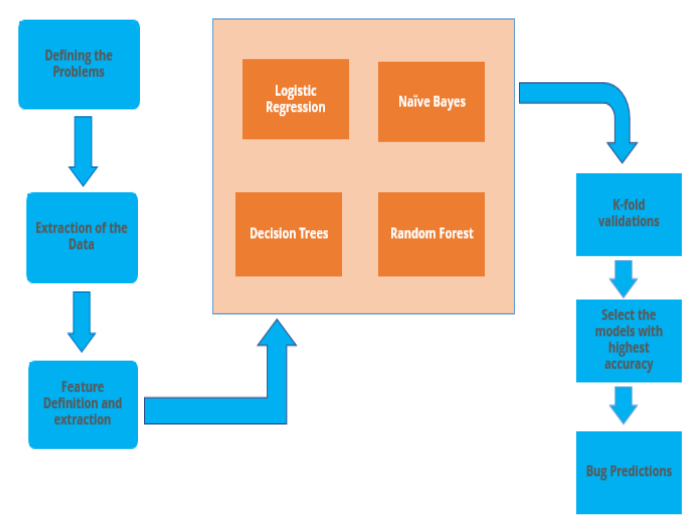

Fig. 1. System Architecture [1]

\subsection{Machine Learning Model:}

The model is simply a set of assumptions about dialysis biomarkers expressed in a probabilistic format. Starting with regression, a supervised learning model, the goal of regression is to predict the value of one or more continuous target variables $b$ given the value of a $\mathrm{D}$-dimensional vector $\mathrm{d}$ of input variables.

\subsection{Machine Learning Parameters:}

We start with a data set that is already labelled with biomarkers values (e.g., clearance). Given all the data sensors and other personal data (age, gender, race, height, smoking etc) expressed as $\mathrm{N}$ observations $\mathrm{dn}$, where $n=1 \ldots N$, with corresponding target values bn, the model tries to learn a pattern that describes how much biomarkers value $b$ are from a new data.

\subsection{Machine Learning Learner:}

These different types of information can be costly to process. With learner, we focus on processing data with machine learning algorithm at real-time.

Models constructed using individual classifiers are tend to have less accuracy. Models are not validated across different samples. Hence, if random samples are taking $\mathrm{n}$, there is high chance of getting less accuracy. Enough priorities were not given for below techniques which can improve the accuracy of the models to the great extent-

1) Variable selection (Prioritization of Factors)

2) Feature engineer and Variable transformations

3) Variable reductions

The proposed structure attempts to resolve the risk issues of ESRD. Solving the problem starts with identifying the right factors required for bug prediction. The prioritized factors are considered for creating analytical data set. As the ensemble classifiers are used, the model becomes a better choice for predictions. Validating the models with K-Fold cross validation technique results in the model effectively working for any kind of data. The proposed system will provide the following benefits- 1) Less Variance and Bias error 2) Better choice of variables through feature engineering and prioritization matrix and 3) High Accuracy for predicting the ESRD risk for new patients.[1]

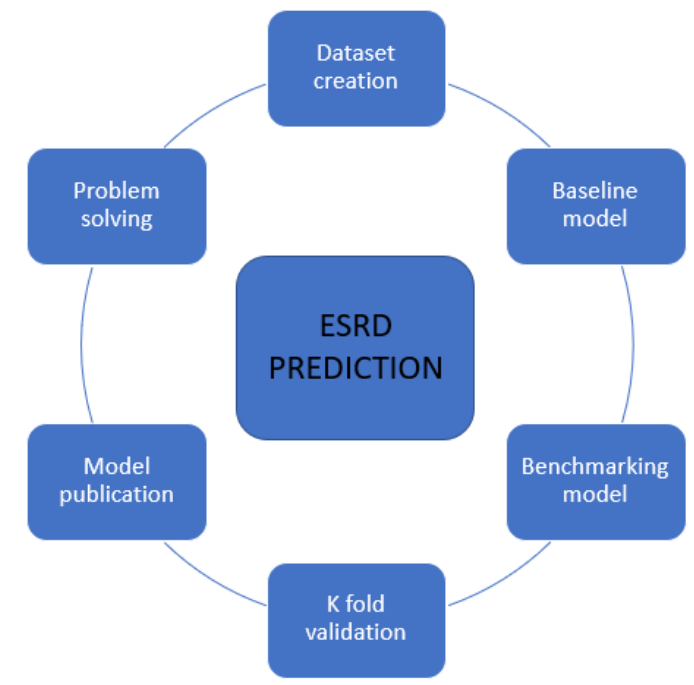

Fig. 2. Machine Learning Model Flow

\subsection{Problem solving framework}

The first step of any machine learning model is understanding the problem and defining the problem better. Extraction of the data from multiple resource and performing operations using various algorithms is done in this step. Steps Involved are:

(1) Defining the problem: Define the current state and need for doing the problem.

(2) Factor Map: List down all the factors required for analyzing the problems.

(3) Hypothesis Generation: Generated hypothesis associated with the problems statement. 
(4) Prioritization Matrix: Identify the factors that are based on actionability and feasibility matrix which leads to prioritize important factors.

(5) Important Factors: Prioritization matrix will help in analyzing the problems associated with the matrix.

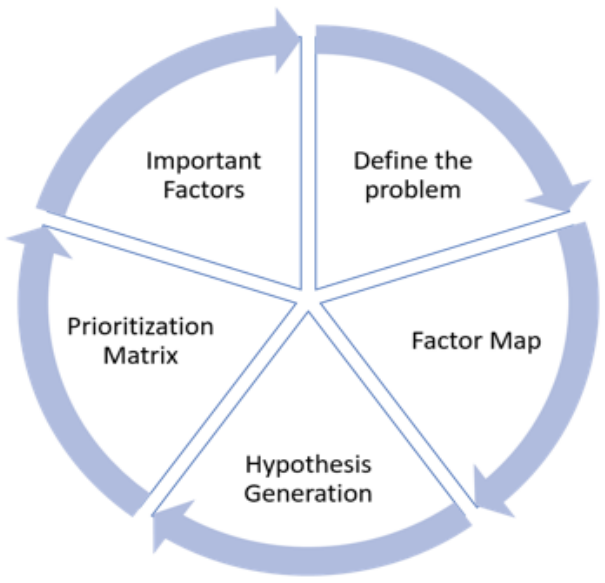

Fig. 4. Problem Solving steps

\subsection{Data preprocessing (Exploratory Data Analysis} or EDA)

Data requires pre-processing which will help to get the right predictions. Propose System involved the below process of exploratory data analysis in sequential order:

1. Data Collection: Collecting the data from multiple resources.

2. Data Merging: Join the data from multiple tables.

3. Null Value Treatment: Treat the null values either by dropping them or by filling them using measures of central tendency, forward fill, backward fill method.

4. Outlier Treatment: Treating the outliers that is not following the interquartile range properties.

5. Garbage Value Treatment: Eliminating the garbage value characters from the datasets.

6. Special Character Removal: Eliminating the various special characters from the datasets.

7. Final Dataset: Creating the analytical ready data set.

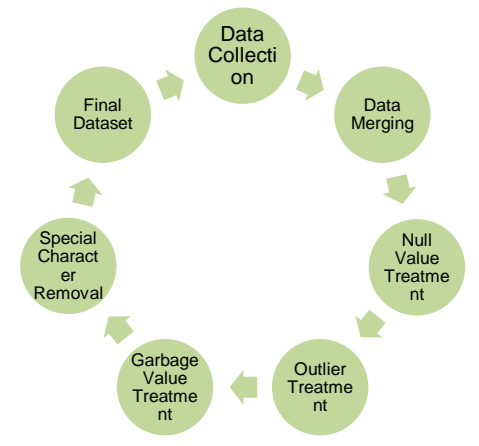

Fig. 3. Representation of Data preprocessing steps

\subsection{Baseline model}

Logistic regression is used as the baseline model. It is more likely to the Ordinary Least Squares (OLS) regression. As the bug prediction is a binary response variable, the bug behavior can be predicted by multiple algorithms. Logistic Regression fits an Sshaped curve to the data. The following steps can be done in Logistic Regression:

(1) Analytical dataset creation: Create the base data set required for the analysis.

(2) Train test split: Dividing the dataset in train and test for executing ML algorithms.

(3) Baseline model's creation: Construct the baseline models with the given set of variables.

(4) Validating the performance of the models: Identify the Accuracy score, Confusion Matrix, Roc- AUC curve designing, Probability curve, Improving model performance and Defect Detection models.

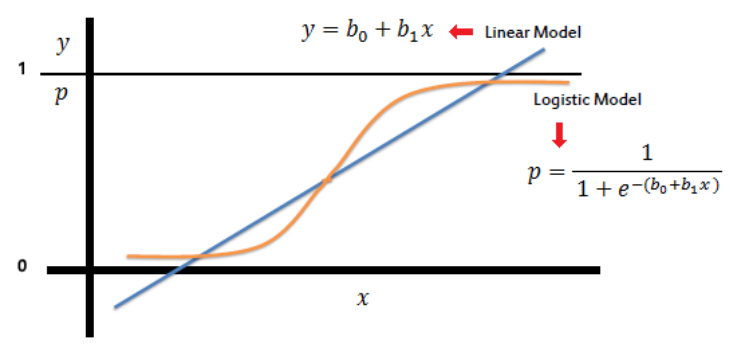

Fig. 5. Logistic Regression curve

\subsection{Benchmarking models}

\subsubsection{KNN}

K-nearest neighbors' algorithm (k-NN) is a nonparametric method used for classification and regression. In both cases, the input consists of the $\mathrm{k}$ closest training examples in the feature space. The output depends on whether $\mathrm{k}-\mathrm{NN}$ is used for classification or regression.

In k-NN regression, the output is the property value for the object. This value is the average of the values of its $\mathrm{k}$ nearest neighbors.

\subsubsection{Random Forest}

Decision tree is also a supervised learning model with pre-defined target variable commonly used in classification of the problems. Decision tree works with the variables which has categorical and continuous input or output. In this method, the inhabitants are grouped into two or more homogeneous sets depending on most substantial differentiator in input variables. How the decision tree will be constructed: There are 4 main criteria's that will decide the how the decision tree will be constructed.[2]

1. Information gain

2. Entropy

3. CHAID score 
International Journal of Research in Advent Technology, Vol.7, No.4, April 2019

E-ISSN: 2321-9637

Available online at $w w w . i j r a t . o r g$

\section{Gini index}

The origin of the decision tress is decided based on the above values. The root node starts with the one that has the highest information gain / entropy.

\subsubsection{Nä̈ve Bayes}

Naive Bayes classifiers are a collection of classification algorithms based on Bayes' Theorem. It is not a single algorithm but a family of algorithms where all of them share a common principle, i.e. every pair of features being classified is independent of each other. Naive Bayes system model is simple to build and predominantly suitable for very huge data sets. Along with effortlessness and simplicity, Naive Bayes is well known for outperforming highly erudite classification methods.

Bayes theorem presents an easy method of calculating posterior probability $\mathrm{P}(\mathrm{c} \mid \mathrm{x})$ from $\mathrm{P}(\mathrm{c}), \mathrm{P}(\mathrm{x})$ and $\mathrm{P}(\mathrm{x} \mid \mathrm{c})$. The equation is represented below:

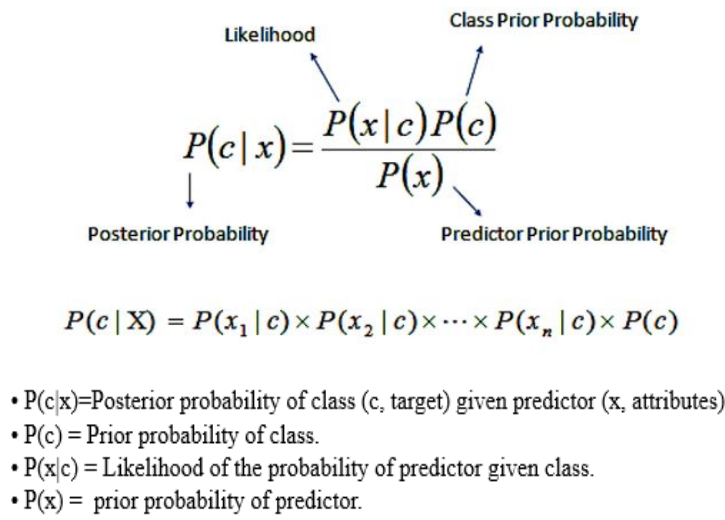

\subsubsection{Ensemble Techniques}

Ensemble methods, which combines several decision trees to produce better predictive performance than utilizing a single decision tree. The main principle behind the ensemble model is that a group of weak learners come together to form a strong learner.

Bagging: Bagging (Bootstrap Aggregation) is used when our goal is to reduce the variance of a decision tree. Here idea is to create several subsets of data from training sample chosen randomly with replacement. Now, each collection of subset data is used to train their decision trees. As a result, we end up with an ensemble of different models. Average of all the predictions from different trees are used which is more robust than a single decision tree.

Boosting: The term 'Boosting' refers to a family of algorithms which converts weak learner to strong learners. To convert weak learner to strong learner, we'll combine the prediction of each weak learner using methods like: 1) Using average or the weighted average 2) Considering prediction has higher vote. XGBoosting: XGBoost is an ensemble learning method. Sometimes, it may not be sufficient to rely upon the results of just one machine learning model.
Ensemble learning offers a systematic solution to combine the predictive power of multiple learners. The resultant is a single model which gives the aggregated output from several models. The models that form the ensemble, also known as base learners, could be either from the same learning algorithm or different learning algorithms. Bagging and boosting are two widely used ensemble learners. Though these two techniques can be used with several statistical models, the most predominant usage has been with decision trees.

\subsection{Model Validation}

\subsection{1 $\mathrm{K}$ fold validation}

$\mathrm{K}$-Fold Cross Validation method is a common type of cross validation technique that is widely used in machine learning.

The general procedure is as follows:

1) Shuffle the dataset randomly.

2) Split the dataset into k groups.

3) For each unique group, take the group as a hold out or test data set.

4) Take the remaining groups as a training data set.

5) Fit a model on the training set and evaluate it on the test set.

6) Retain the evaluation score and discard the model.

7) Summarize the skill of the model using the sample of model evaluation scores.

\subsubsection{Grid search CV}

Grid search technique is used to find the best combination of hyper-parameters for a given model and test dataset. In this scenario, there are several models, each with a different combination of hyperparameters. Each of these combinations of parameters, which correspond to a single model, can be said to lie on a point of a "grid". The goal is to train each of these models and evaluate them using cross-validation. Selection is done based on the performance.

\section{CONCLUSION}

Machine Learning Application in End-Stage Renal Disease Risk Prediction or ESSRD risk prediction requires various connected objects and these are the main keys for various intelligent structures for illustration. This paper intents to develop a noninvasive approach that predict various risks of dialysis patient in End-Stage Renal Disease (ESRD). In this paper, we propose various machine learning techniques to build a model for gathering various data under the data analytics environment which can predict the occurrence of ESRD disease by using the ESRD data generated from various IOT Sensors [1]. We have used Naïve Bayes, Random Forest and KNN classifier-based classification models and Bagging, Boosting, XGBoosting techniques are used for improving the overall accuracy of the classifier in the classification ESRD risk. For the same we apply preprocessing techniques so that accurate data is fed as 
an input to the training process, our proposed approach classify the ESRD risk as Positive and Negative and helps in faster decision making by the doctors. The work of proposed model has gone through preprocessing stage and classifiers learning stage. For analytical evaluation of the proposed classifier accuracy and f-measure are used. The comparative results prove that hybrid model improved the overall classification accuracy and f-measure of ESRD risk prediction as compared to traditional existing techniques for classification.

\section{REFERENCES}

[1] Nipa Sarkar, and Asha Rani Borah." End-Stage Renal Disease Risk Prediction via Machine Learning and IoT", International Journal of Research in Advent Technology, Vol.6, No.11, November 2018

[2] Dileep Kumar G "Chapter-1 Tree based modelling techniques: IGI GLOBAL,2019.

[3] Dejun Mu, Junhong Duan, Xiaoyu Li,Hang Dai, Xiayan Cai, Latin Guo. "Expede Herculem: Learning Multi Labels from Single Label”, IEEE Acess, 2018.

[4] Roy, Sandip Kumar, and Preeta Sharan. "Application of Machine Learning For Real-time Evaluation of Salinity (or TDS) in Drinking Water Using Photonic Sensor", Drinking Water Engineering and Science Discussions, 2016.

[5] Han Feng. "The application of artificial intelligence in electrical automation control", Journal of Physics: Conference Series, 2018.

[6] The International Conference on Advanced Machine Learning Technologies and Applications(AMLTA2018)", Springer Nature, 2018.

[7] Iman Raeesi Vanani, Morteza Amirhosseini. "Chapter 3 Deep Learning for Opinion Mining", IGI Global, 2019. 